\title{
Aberration-Correction in a Monochromated and Energy Filtered Environment
}

\author{
D.C. Bell ${ }^{*}$, S. Meyer ${ }^{* *}$, A. Orchowski* ${ }^{* *}$ P. Tayebati** and G. Benner ${ }^{* *}$ \\ *School of Engineering and Applied Sciences, Harvard University, Cambridge, MA 02138 \\ ${ }^{* *}$ Carl Zeiss NTS GmbH, Carl Zeiss SMT, Oberkochen Germany
}

The history of electron microscope development shows that the trend in electron gun design has been towards brighter emission with less energy spread [1]. Thermal emission yields energy spreads in the order of $1.2 \mathrm{eV}$ or so, the Schottky FEG $0.8 \mathrm{eV}$ and cold FEG's down to the 0.3 $\mathrm{eV}$ region. The competing needs for high brightness, cost and stability have meant that cold FEG's are not as commonly available from microscope vendors as the thermal FEG. In the development process to produce an electron source with all the benefits of both cold and thermal emitters the incorporation of a monochromator on a thermal source is one of the mechanisms of choice to minimize the energy spread.

Objective lens Cs correction on a Schottky FEG system has many well known and demonstrated advantages over non-corrected electron microscopes both for TEM and STEM applications. The combination of Cs correction and a FEG source monochromator yields a further step along the path to an ideal microscope, the expansion of the temporal envelope function of the contrast transfer function by reducing the delta $\mathrm{E}$ is a direct benefit that can lead to improved resolution (Figure 1). The further benefits of monochromator integration are for applications that especially depend on energy resolution such as spectroscopy and advanced contrast mechanisms such as "atomic scale" energy filtered imaging.

Significant improvements have been made to the standard Zeiss Libra 200 FE platform for installation at Harvard University including frame stability, power supplies, the addition of a second generation Omega monochromator and a corrected Omega in-column energy filter, Figure 2. For demonstration of the achieved information transfer, Young's fringes patterns (as exemplified and measured in Figure 3) have been generated from micrographs recorded. The energy spread of the field emission source was reduced by the monochromator to $0.2 \mathrm{eV}$ and a residual spherical aberration of the objective lens (Cs value) of approximately $-3 \mu \mathrm{m}$ was obtained using the integrated aberration corrector from CEOS $\mathrm{GmbH}$. An amorphous Tungsten thin film was used as specimen. The advantages of the addition of an energy filter allows for the ability to image only the elastically scattered electrons for HRTEM imaging (or the inelastically scattered if needed) and provides an additional mechanism for contrast.

Tunable Cs correction, monochromator and in-column energy filtering combined on one instrument platform provide a foundation for the future with a high resolution transmission electron microscope with advanced resolution and contrast capabilities.

References

[1] P.W. Hawkes and J.C.H. Spence Science of Microscopy Springer (2007) 

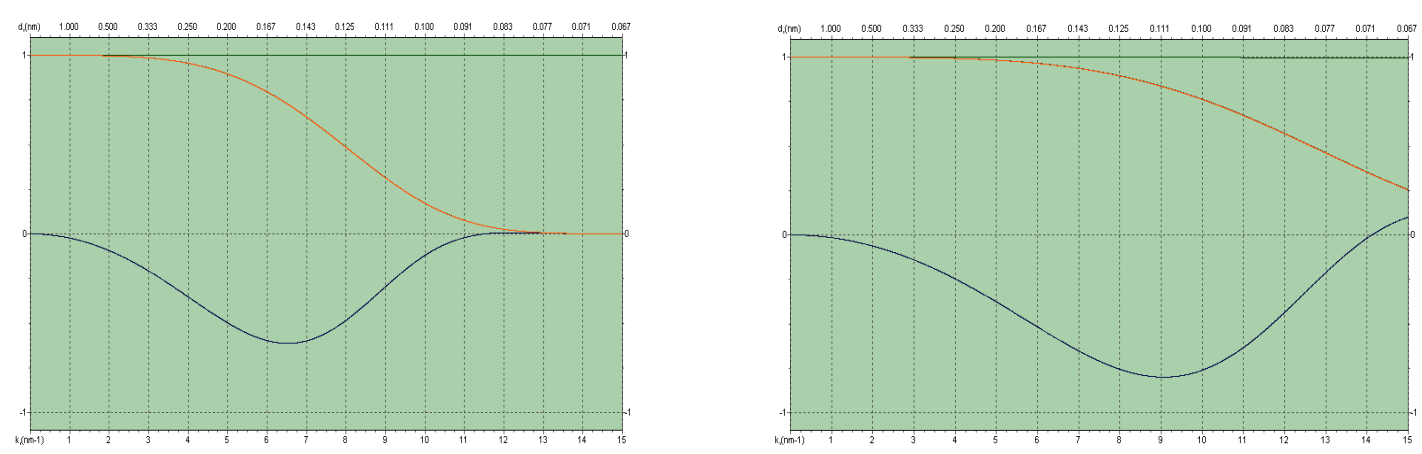

Figure 1. Left, Contrast Transfer function of 200KV Cs corrected transmission electron microscope. Right, Contrast Transfer Function of 200KV Cs TEM with monochomator allowing $0.2 \mathrm{eV}$ beam energy spread.
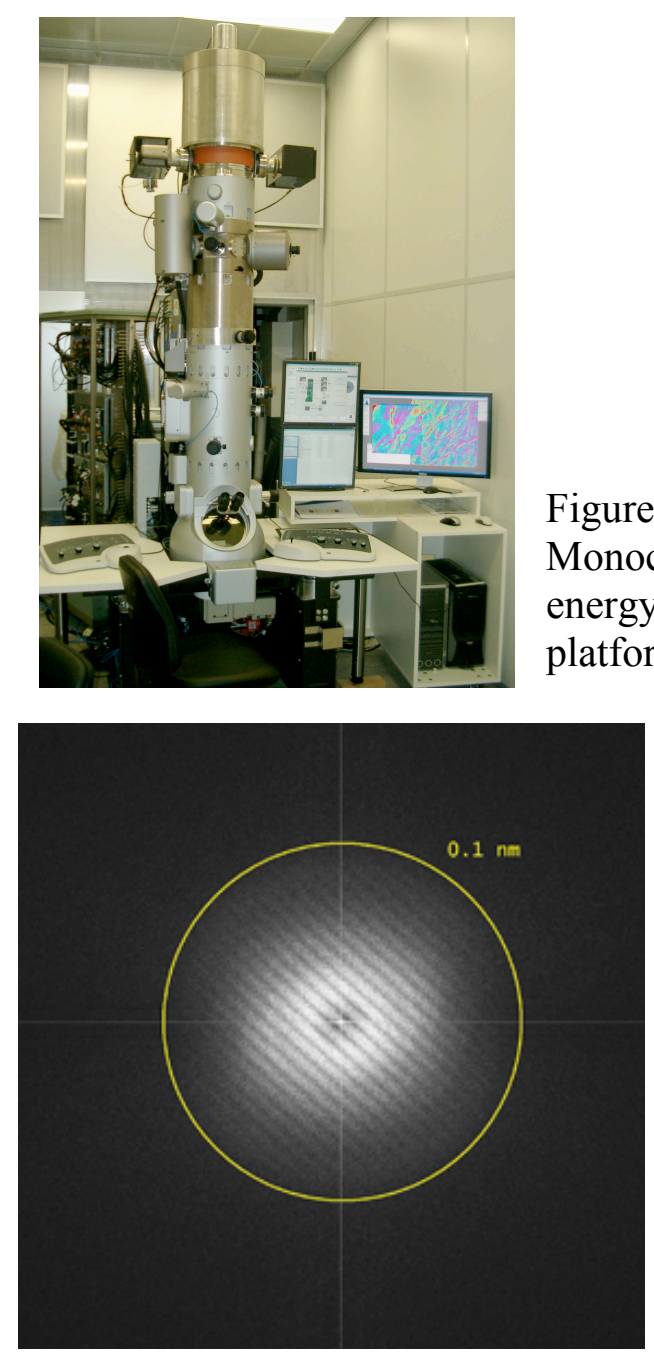

Figure 2.

Monochromated, aberration-corrected TEM with in-column energy filter, as developed from the Zeiss Libra $200 \mathrm{MC}$ platform

Figure 3. Left, Young's fringes produced at 200KV from amorphous Tungsten film with Cs correction showing information transfer to slightly better than $0.1 \mathrm{~nm}$.

Right, Young's fringes produced with the same Cs correction but with monochromation selection of $0.2 \mathrm{eV}$ energy spread indicating an information transfer to $0.073 \mathrm{~nm}$. 